\title{
SIGNO RADIOLOGICO: LAS VALIOSAS PERLAS DEL INTESTINO DELGADO
}

\author{
Drs. Mario Zerega $\mathbf{R}^{(1)}$, Claudio Cortés $A\left({ }^{2}\right.$.
}

1. Médico Cirujano, Becado de Radiología Universidad de Chile.

2. Médico Radiólogo, Universidad de Chile, Universidad del Desarrollo.

\section{THE VALUABLE PEARLS OF THE SMALL INTESTINE}

\begin{abstract}
The string of pearls sign can be seen in simple abdominal radiography and computed tomography as well. It consists of a row of small air bubbles longitudinally oriented in the abdomen. They represent small amounts of air trapped between valvulae conniventes along the non dependent wall of predominantly fluid-filled, dilated small bowel loops. This sign is considered virtually diagnostic of small bowel obstruction, which occurs whenever there is an intrinsic or extrinsic blockage of the normal flow of bowel content and manifests as abdominal pain, distention, vomiting, and increased high-pitched bowel sounds. It is a potentially life threatening disease that requires urgent treatment. Duration of obstruction, vomiting frequency and gastric or enteric suction may affect radiographic appearance. It continues being hard to diagnose only based on simple radiography. Despite its low frequency, the string of pearls sign could be very valuable for radiologist, clinical physician and patient.

Keywords: Intestinal obstruction, Small bowel, String of pearls.
\end{abstract}

Resumen: El signo del collar de perlas puede ser visto en radiografía simple de abdomen y en tomografía computada. Consiste en un conjunto de pequeñas burbujas dispuestas en forma longitudinal. Representan pequeños acúmulos de gas atrapados entre las válvulas conniventes de asas de intestino delgado dilatadas y predominantemente rellenas de líquido a lo largo de su pared no dependiente ${ }^{(1)}$. Es considerado virtualmente diagnóstico de obstrucción intestinal, que ocurre cuando existe un bloqueo intrínseco o extrínseco al flujo normal del contenido intestinal que se manifiesta con dolor abdominal, distensión,

Zerega M y cols. Las valiosas perlas del instestino delgado. Rev Chil Radiol 2009; 15(4): 202-205. Correspondencia: Dr. Mario Zerega $R$. mariozeregaruiz@gmail.com

Trabajo recibido el 25 de septiembre de 2009, aceptado para publicación el 10 de noviembre 2009. vómitos, borborigmos y bazuqueo. Es una enfermedad potencialmente letal que requiere ser resuelta en forma urgente. La duración de la obstrucción, la frecuencia de los vómitos, y la aspiración del contenido gástrico o enteral pueden afectar la apariencia radiográfica. Continúa siendo difícil de diagnosticar solamente con radiografía simple de abdomen. Aunque es poco frecuente, el signo del collar de perlas es muy valioso para el radiólogo, el clínico y el paciente.

Palabras clave: Collar de perlas, Intestino delgado, Obstrucción intestinal.

\section{LAS VALIOSAS PERLAS DEL INTESTINO DELGADO}

Aspecto Imaginológico

El signo del collar de perlas (Figura 1), en la literatura anglosajona "string of pearls" o "string of beads"(1), puede ser visto en radiografía simple de abdomen en posición decúbito supino, decúbito lateral con rayo horizontal (Figura 2), de pie (Figura 3) y en tomografía computada (2) (Figura 4). Consiste en un conjunto de burbujas dispuestas en forma longitudinal, adyacentes unas a otras. Representan pequeños acúmulos de gas atrapados entre las válvulas conniventes a lo largo de la pared superior

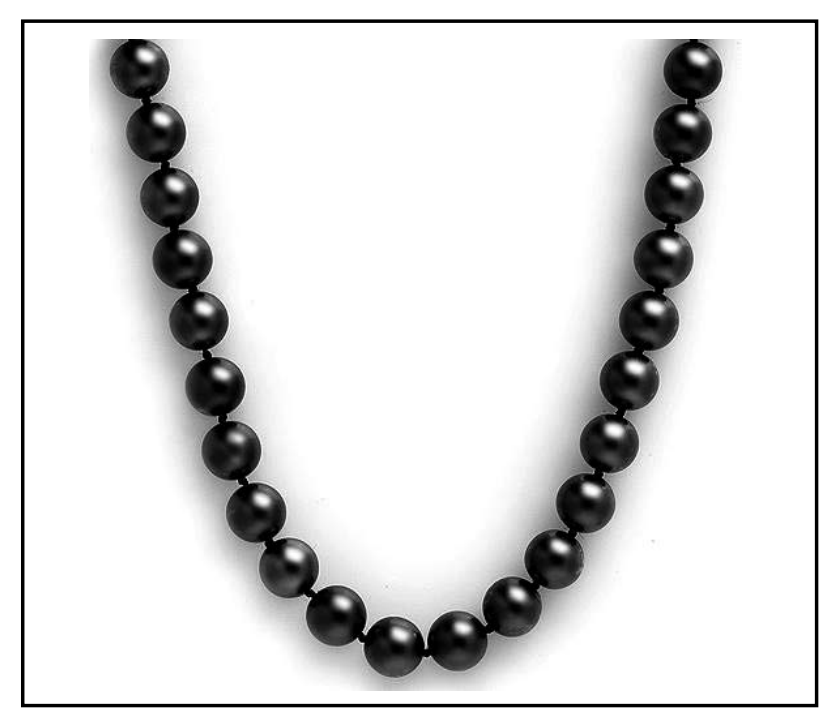

Figura 1. Collar de perlas negras. 
o anterior de asas de intestino delgado dilatadas y predominantemente rellenas de líquido, a lo largo de su pared superior ${ }^{(1)}$. Este signo es visible gracias a las diferentes densidades del gas y del líquido en contacto.

También ha sido descrita con el mismo nombre la apariencia arrosariada del conducto pancreático en casos de pancreatitis crónica, visualizada mediante colangio pancreatografía endoscópica retrógada ${ }^{(3)}$, y de la arteria mesentérica superior $o$ arterias renales en casos de displasia fibromuscular, visualizadas en angiografía ${ }^{(4)}$. Nos referiremos al signo descrito en intestino delgado ${ }^{(5)}$.

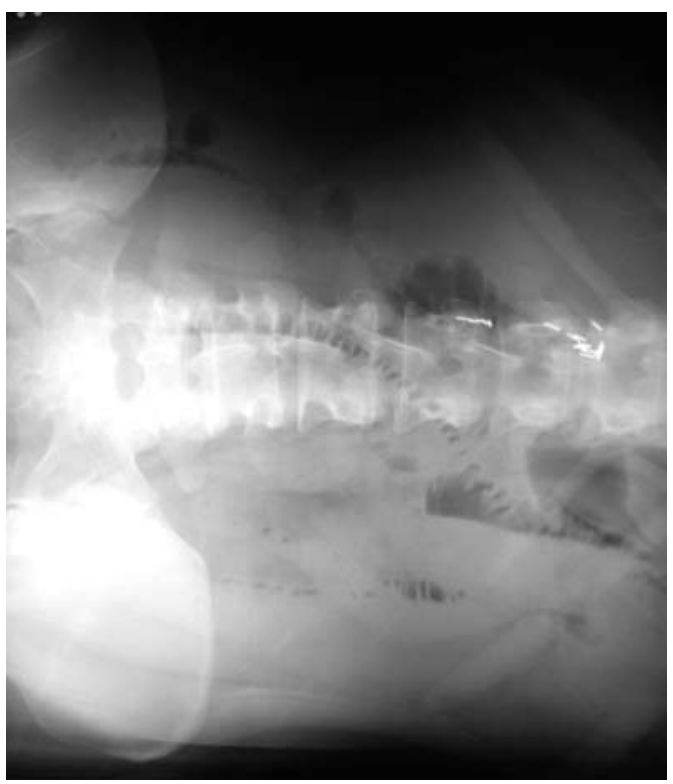

Figura 2. Radiografía simple de abdomen en decúbito lateral izquierdo, que muestra múltiples burbujas de gas dispuestas en un trayecto longitudinal (signo del collar de perlas) en una paciente con obstrucción intestinal.

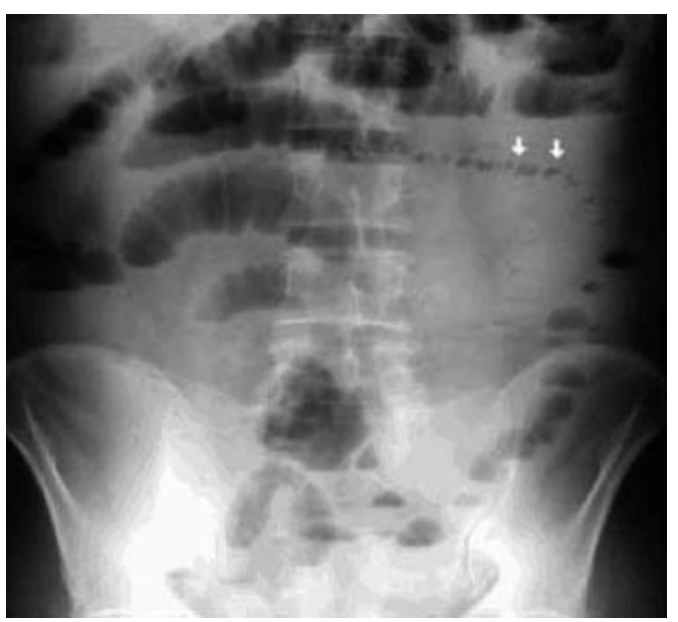

Figura 3. Radiografía simple de abdomen en posición de pie, que muestra múltiples niveles hidroaéreos y el signo del collar de perlas (flechas) [reproducido de Cortés $C$. Gases del abdomen su utilidad diagnóstica: gases endoluminales (segunda parte). Rev Chil Radiol 2002; 8(1): 107-18].

\section{Significado}

En un contexto clínico adecuado, este signo es considerado virtualmente diagnóstico de obstrucción enteral. La obstrucción mecánica del intestino delgado ocurre cuando existe un bloqueo intrínseco o extrínseco al flujo normal del contenido intestinal. Los síntomas y signos clásicos de obstrucción intestinal incluyen: dolor abdominal, distensión, borborigmos (ruidos abdominales aumentados de timbre agudo) y bazuqueo (provocación de borborigmos al movilizar el abdomen del paciente). Representa una causa frecuente de abdomen agudo, dando cuenta de aproximadamente el $20 \%$ de las cirugías de urgencia por este síndrome. Las bridas o adherencias postquirúrgicas son su causa más frecuente, que representan entre 50 y $75 \%$ de los casos, seguidas por las hernias, con aproximadamente $10 \%$, y tumores malignos con menor frecuencia (generalmente metástasis) ${ }^{(6-8)}$. Para efectos prácticos, estas tres causas dan cuenta del $80 \%$ de los $\operatorname{casos}^{(9)}$.

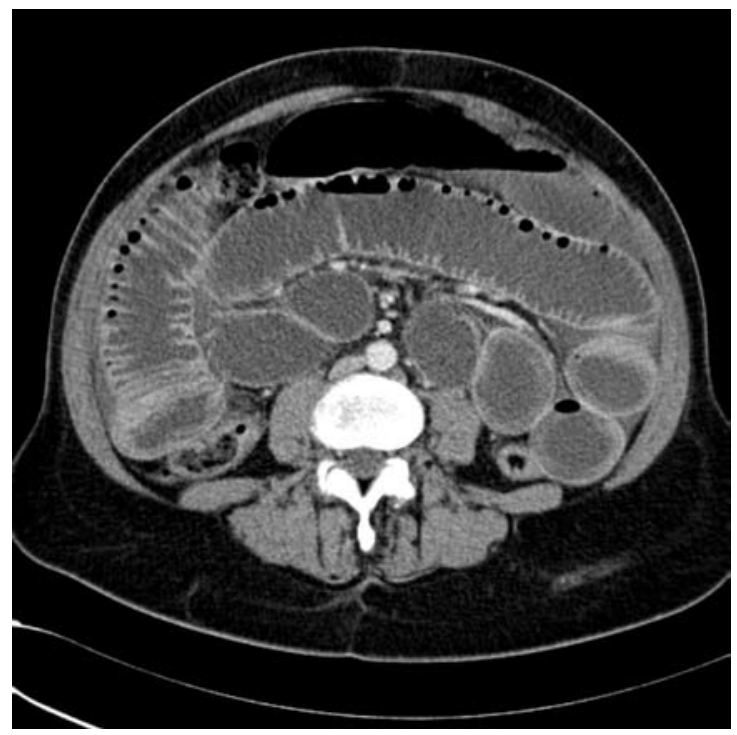

Figura 4. Corte axial de tomografía computada de abdomen y pelvis que muestra asas de intestino delgado dilatadas predominantemente rellenas de líquido, con burbujas de gas atrapadas entre las válvulas conniventes de su pared ventral (signo del collar de perlas). Se observa una cicatriz de laparotomía media por una obstrucción intestinal por bridas.

\section{Diagnóstico diferencial}

Aunque con menor frecuencia, también puede ser visto en casos de:

- Gastroenteritis aguda: el cuadro clínico es diferente ya que el paciente presenta diarrea.

- lleo paralítico: el paciente presenta enlentecimiento o ausencia de flujo intestinal, pero no hay una disminución abrupta de calibre entre asas dilatadas y colapsadas (no hay obstrucción mecánica). Puede ser difícil distinguirlo de obstrucción intestinal en radiografía simple. 


\section{Discusión}

La obstrucción intestinal continúa siendo difícil de diagnosticar solamente sobre la base de la radiografía simple de abdomen. La duración de la obstrucción, la frecuencia de los vómitos y la aspiración del contenido gástrico o enteral pueden afectar la apariencia radiográfica ${ }^{(5)}$. La radiografía con rayo horizontal y la radiografía en decúbito supino, son los exámenes iniciales de elección cuando se sospecha el diagnóstico de obstrucción intestinal. Mirvis y cols. encontraron que la radiografía de pie no agregaba información adicional a la radiografía en decúbito en pacientes con obstrucción intestinal demostrada ${ }^{(10)}$. De hecho, el paciente con sospecha de obstrucción intestinal presenta dolor abdominal intenso, compromiso de su estado general y está invadido por vías venosas, lo que al intentar ponerlo de pie dificulta su movilización, hace más demorosa la toma de la radiografía y aumenta la tasa de repeticiones por movimiento.

Las asas de intestino delgado dilatadas usualmente pueden ser reconocidas dentro de las 3 a 5 primeras horas desde el inicio de la obstrucción. Aunque imágenes secuenciales en un lapso de 12 a 24 horas son útiles para establecer un diagnóstico evolutivo de un cuadro obstructivo, con la disponibilidad actual de imágenes seccionales, es inusual esperar ese plazo.

Se han reportado eficacias similares en la detección de obstrucción intestinal de distinta severidad con radiografía convencional y tomografía computada ${ }^{(11)}$. Sin embargo, la tomografía computada permite más frecuentemente identificar la causa de la obstrucción, y otorga información adicional para decisiones terapéuticas $^{(12)}$ (Figuras 5 y 6 ).

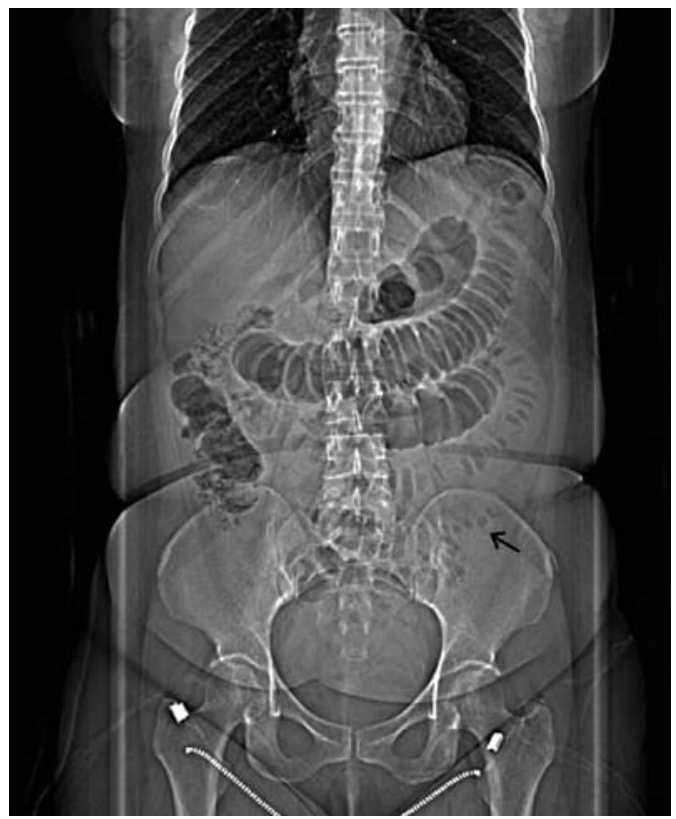

Figura 5. Topograma de tomografía computada de abdomen y pelvis que muestra asas de intestino delgado dilatadas con gas en su interior y signo del collar de perlas (flecha).

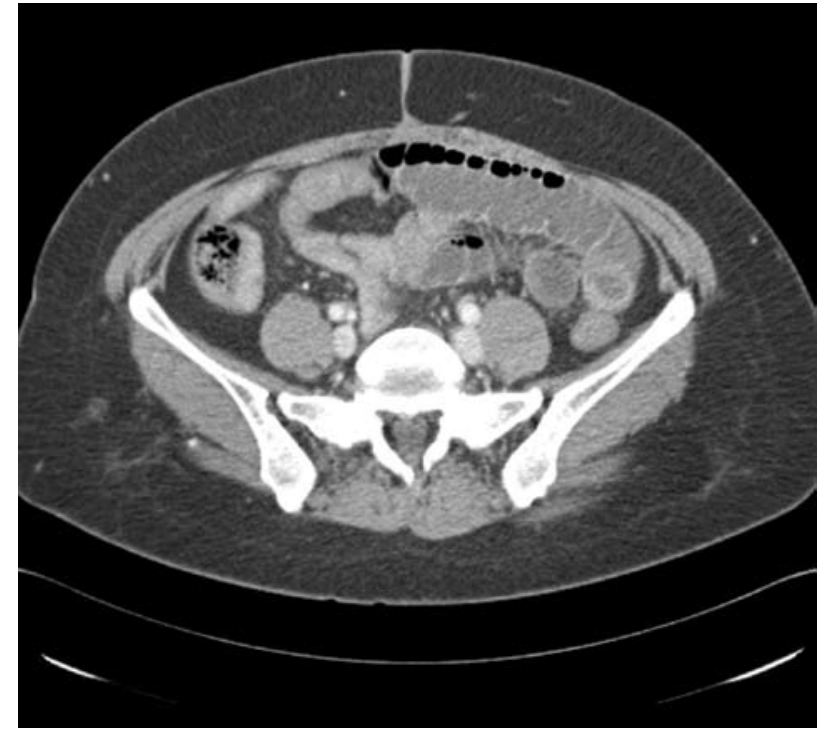

Figura 6. Corte axial de tomografía computada de abdomen y pelvis del mismo paciente de la figura 4, que demuestra obstrucción por bridas atribuible a una laparotomía previa. Proximal al punto de colapso se observa el signo del collar de perlas.

La obstrucción del tránsito intestinal determina acumulación de contenido líquido y gas. El intestino se dilata y el peristaltismo es más intenso en un esfuerzo por propulsar el contenido hacia distal, lo que se traduce en dolor tipo cólico y borborigmos. Mientras más distal sea la lesión obstructiva, más asas estarán distendidas. Así, el intestino distendido contiene cantidades variables de gas y líquido, lo que explica los niveles hidroaéreos vistos en las radiografías con rayo horizontal. Existe controversia acerca de su importancia. En un estudio reciente, el hallazgo de más de dos niveles hidroaéreos en asas de intestino delgado dilatadas, niveles hidroaéreos a diferentes alturas dentro de la misma asa, y niveles de más de $2,5 \mathrm{~cm}$ de ancho fueron considerados indicativos de obstrucción ${ }^{(13)}$. Sin embargo, otros autores ${ }^{(14-16)}$ han reportado que estos hallazgos son frecuentes en casos de íleo adinámico y por ende son un indicador poco confiable de obstrucción intestinal.

Hay que recordar que el gas intestinal en asas obstruidas puede absorberse y eliminarse durante los vómitos, y disminuye a medida que transcurre más tiempo desde el inicio de la obstrucción. No debe esperarse la existencia de niveles hidroaéreos. Después de la etapa del collar de perlas, las asas obstruidas sólo contienen líquido y, si el signo fundamental es la detección de niveles hidroaéreos el paciente morirá antes de que hagamos el diagnóstico correcto ${ }^{(17)}$.

Thompson y cols. detectaron el signo del collar de perlas en sólo 3 (10\%) de 29 pacientes con obstrucción intestinal, y en $1(1,7 \%)$ de 61 pacientes sin obstrucción. Concluyeron que aunque el signo del collar de perlas es poco frecuente en los pacientes 
con obstrucción intestinal, su presencia o ausencia son discriminativas para la inclusión o exclusión del diagnóstico, respectivamente ${ }^{(13)}$. Aunque no hay reportes en la literatura de la duración o temporalidad de este signo, probablemente su presencia sea transitoria, de corta duración y relativamente tardía dentro de la evolución del cuadro obstructivo.

La mayor parte del gas proximal al punto de obstrucción es aire deglutido. Así, si los pacientes con obstrucción tragan poco o nada de aire, por ejemplo en caso de acalasia, entonces la radiografía abdominal supina puede no demostrar distensión aérea. Las radiografías de pie o en decúbito podrían no demostrar niveles hidroaéreos ni signo del collar de perlas.

\section{Comentario}

En conclusión, las perlas negras del intestino delgado son escasas pero muy valiosas para el radiólogo, para el clínico y para el paciente. El signo del collar de perlas nos permite diagnosticar a tiempo una enfermedad grave, potencialmente letal sin tratamiento.

\section{Bibliografía}

1. Nevitt PC. The string of pearls sign. Radiology. 2000; 214(1): 157-158.

2. Cortés C. Gases del abdomen su utilidad diagnóstica: gases endoluminales (primera parte). Rev Chil Radiol 2002; 8(1): 5-12.

3. Balthazar EJ. Pancreatitis. In: Gore RM, Levine MS, Laufer I, editors. Textbook of gastrointestinal radiology. First ed. Philadelphia: WB. Saunders Co. 1994; p. 2132-2160.

4. Nemcek AA. Angiography and interventional radiology. In: Gore RM, Levine MS, Laufer I, editors. Textbook of gastrointestinal radiology. First ed. Philadelphia: WB. Saunders Co. 1994; p. 802-817.

5. Messner JM. Gas and soft tissue abnormalities. In: Gore RM, Levine MS, Laufer I, editors. Textbook of gastrointestinal radiology. First ed. Philadelphia: WB.
Saunders Co. 1994; p. 169-192.

6. Mucha P, Jr. Small intestinal obstruction. Surg Clin North Am. 1987; 67(3): 597-620.

7. Miller G, Boman J, Shrier I, Gordon PH. Etiology of small bowel obstruction. Am J Surg 2000; 180(1): 3336.

8. Furukawa A, Yamasaki M, Furuichi K, Yokoyama K, Nagata T, Takahashi M, et al. Helical CT in the Diagnosis of Small Bowel Obstruction. Radiographics. 2001; 21(2): 341-355.

9. Maglinte DD, Balthazar EJ, Kelvin FM, Megibow AJ. The role of radiology in the diagnosis of small-bowel obstruction. AJR Am J Roentgenol. 1997; 168(5): 11711180.

10. Mirvis SE, Young JW, Keramati B, McCrea ES, Tarr R. Plain film evaluation of patients with abdominal pain: are three radiographs necessary? AJR Am J Roentgenol 1986; 147(3): 501-503.

11. Maglinte DD, Reyes BL, Harmon BH, Kelvin FM, Turner WW, Jr., Hage JE, et al. Reliability and role of plain film radiography and CT in the diagnosis of small-bowel obstruction. AJR Am J Roentgenol. 1996; 167(6): 1451-1455.

12. Silva AC, Pimenta M, Guimaraes LS. Small Bowel Obstruction: What to Look For1. Radiographics. 2009 March 2009;29(2):423-39.

13. Thompson WM, Kilani RK, Smith BB, Thomas J, Jaffe TA, Delong DM, et al. Accuracy of Abdominal Radiography in Acute Small-Bowel Obstruction: Does Reviewer Experience Matter? Am J Roentgenol. 2007; 188(3): W233-238.

14. Gammill SL, Nice CM, Jr. Air fluid levels: their occurrence in normal patients and their role in the analysis of ileus. Surgery. 1972; 71(5): 771-780.

15. Levin B. Mechanical small bowel obstruction. Semin Roentgenol 1973; 8(3): 281-297.

16. Harlow CL, Stears RL, Zeligman BE, Archer PG. Diagnosis of bowel obstruction on plain abdominal radiographs: significance of air-fluid levels at different heights in the same loop of bowel. AJR Am J Roentgenol. 1993; 161(2): 291-295.

17. Cortés $\mathrm{C}$. Gases del abdomen su utilidad diagnóstica: gases endoluminales (segunda parte). Rev Chil Radiol 2002; 8(1): 107-118. 